\title{
UN NUEVO ELEUTHERODACTYLUS (ANURA, LEPTODACTYLIDAE) DE LA CORDILLERA DE MÉRIDA, ANDES DE VENEZUELA
}

\author{
C. L. Barrio-Amorós* y A. Chacón-Ortiz**
}

\begin{abstract}
RESUMEN
Se describe una nueva rana del género Eleutherodactylus de la Cordillera de Mérida, Andes de Venezuela. La especie se distingue del resto de congéneres por su tamaño moderado, primer dedo de la mano igual o más corto que el segundo, palmeadura pedal basal, y color dominante marrón uniforme o con pocas manchas. Se compara la especie con los grupos unistrigatus y conspicillatus, pero no se asigna a ninguno de ellos. Se presenta un mapa de distribución y un sonograma del canto. Se comenta brevemente sobre el conocimiento actual de las ranas del género Eleutherodactylus en Venezuela.

Palabras clave: Anura, Leptodactylidae, Eleutherodactylus yustizi nueva especie, Cordillera de Mérida, Andes, Venezuela.
\end{abstract}

\section{ABSTRACT \\ A new Eleutherodactylus (Anura, Leptodactylidae) from the Cordillera de Mérida, Venezuela}

A new frog of the genus Eleutherodactylus is described from the Cordillera de Mérida, Venezuelan Andes. The species is distinguished from others by its moderate size, first finger equal or shorter than second, basal webbing on toes, and dominant colour immaculate brown or with a few spots. The species is compared with the groups unistrigatus and conspicillatus, but not assigned to either of them. A distribution map and a sonogram (of the song) are presented. Comments about current knowledge of frogs of the genus Eleutherodactylus in Venezuela are provided.

Key words: Anura, Leptodactylidae, Eleutherodactylus yustizi new species, Cordillera de Mérida, Andes, Venezuela.

\section{Introducción}

El estado de conocimiento sobre las ranas del género Eleutherodactylus de la Cordillera de Mérida (Venezuela) se encuentra aún en una fase muy preliminar. Sus especies, incluyendo la que aquí se describe como nueva, se han citado en dicha
Cordillera en cinco estados. Según la recopilación de Barrio Amorós (1998), en el estado Táchira se conocen siete especies: E. anolirex Lynch, 1983, E. chlorosoma Rivero, 1984, E. lentiginosus Rivero, 1984, E. melanoproctus Rivero, 1984, E. mondolfii Rivero, 1984, E. prolixodiscus Lynch 1978 y E. pulidoi Rivero, 1984; E. nicefori Cochran et Goin,

* Fundación AndígenA. Apartado Postal 210, 5101-A. Mérida, Venezuela. E-mail: cesarlba@yahoo.com

** Instituto Venezolano de Investigaciones Científicas (IVIC). Altos de Pipe, Km. 11 Carretera Panamericana, vía San Antonio de los Altos, Estado Miranda, Venezuela. Email: aecortiz@yahoo.com 
1971, es conocido del páramo de Tamá, una extensión de la Cordillera Oriental de Colombia en Venezuela. En el estado Mérida se conocen nueve especies: E. briceni (Boulenger, 1903), E. chlorosoma, E. colostichos La Marca et Smith, 1982, E. ginesi (Rivero, 1964), E. lancinii Donoso-Barros, 1968, E. paramerus Rivero, 1984, E. pleurostriatus Rivero, 1984, E. tubernasus Rivero, 1984 y E. vanadisae La Marca, 1984. Tres especies son conocidas en el estado Trujillo: E. boconoensis Rivero et Mayorga, 1973, E. ginesi y E. lancinii (aunque sabemos que la especie que se cuenta como $E$. gine$s i$ es una especie distinta [J. D. Lynch, com. pers., junio 2001] y la que se tiene como E. lancinii, por razones biogeográficas tampoco debe de representar el mismo taxón). Para el estado Lara, Yústiz (1996) menciona tres especies, una de Yacambú, otra de Terepaima y otra de Guariquito, todas por describir; una de ellas (la de Yacambú) es la nueva especie que se describe en este trabajo. En el estado Portuguesa, la primera especie que se cita es también la misma que describimos a continuación. En el ramal andino del estado Zulia (Sierra de Perijá, la cual no integra la Cordillera de Mérida) se conoce solamente una referencia, Rivero (1964), de un Eleutherodactylus, clasificado tentativamente como E. urichi (Boettger, 1894). Como ya aclaran Kaiser et al. (1994), E. urichi es endémica de Trinidad, por lo que la especie citada de Perijá debe ser revisada; lamentablemente, el ejemplar referido (MHNLS 409) se halla en muy mal estado de conservación, que hace imposible una identificación certera. El primer autor está describiendo con Tito Barros otras dos especies de esta serranía. Recientemente La Marca (2004) describe E. pedimontanus del piedemonte andino de la Cordillera de Mérida.

Durante una exploración preliminar de una selva nublada, a $1.400 \mathrm{~m}$ en la vertiente SE de la Cordillera de Mérida, en el estado Barinas, durante noviembre de 1995, se observó una especie de Eleutherodactylus que no concordaba con ninguna descrita; ésta fue fotografiada pero no colectada. El estudio de las colecciones de herpetología de la Facultad de Ciencias, Universidad de los Andes, Mérida (CVULA), del Museo de Biología de la Universidad Central de Venezuela (MBUCV) y del Museo de Biología Rancho Grande, Maracay (EBRG), demostró que varios individuos provenientes de Yacambú y varias localidades del estado Táchira que se encontraban depositados sin identificar, pertenecían a la misma especie. Un reconocimiento del Parque Nacional Yacambú, en las estribaciones de orientación NE de la Cordillera de
Mérida, estado Lara, denotó la presencia de unas hembras de Eleutherodactylus de tamaño considerable, que tampoco fueron colectadas por carecer de permisos. Consultando con Enrique Yústiz, una autoridad en herpetofauna de este estado, la validez de la especie fue confirmada. Tras examinar la serie de animales reunidos para estudio, se comprobó que las hembras de Yacambú y una de Táchira deben ser conespecíficas con los machos de Barinas, Lara y Táchira. Una serie de ejemplares de esta especie colectados por E. Yústiz y previamente depositados en la UCLA (Universidad Centro Occidental Lisandro Alvarado, Barquisimeto), cuya colección se ha perdido en su mayor parte debido a una inundación, fue transferido de esta Universidad a la colección de la CVULA. El propósito de este trabajo es describir esta nueva especie.

\section{Material y métodos}

Las medidas fueron tomadas con un calibrador (hasta $0.1 \mathrm{~mm}$ ). Las abreviaturas de las medidas utilizadas en el texto son: LCC: Longitud cabezacuerpo; LT: Longitud de la tibia; LF: Longitud del fémur ; LP: Longitud del pie; ACa: Anchura cefálica; LCa: Longitud cefálica; DIN: Distancia internasal; APS: Ancho del párpado superior; DIO: Distancia interorbital; DON: Distancia entre la comisura anterior del ojo y la narina; DO: Diámetro del ojo (horizontal); DT: Diámetro del tímpano (horizontal); A3D: Anchura del disco del tercer dedo (mano derecha); A4D: Anchura del disco del cuarto dedo del pie (derecho); LN: Longitud del hocico (desde el borde anterior del ojo hasta la punta del hocico); L1D: Longitud del primer dedo de la mano (derecha); L2D: Longitud del segundo dedo de la mano (derecha). La descripción de la coloración del animal en vida se basa en diapositivas de ejemplares no colectados. El método sigue a Lynch \& Duellman (1997); Duellman (1992) y Rueda y Lynch (1983); la terminología en castellano de las estructuras en manos y pies siguen a Lynch (1999). El sexo se determina por la presencia/ ausencia de aberturas vocales.

Las abreviaturas de las colecciones y acrónimos de los museos son como sigue: CVULA: Colección de Vertebrados, Universidad de los Andes, Mérida, Venezuela; EBRG: Museo de la Estación Biológica Rancho Grande, Maracay, Venezuela; MBUCV: Museo de Biología de la Universidad Central de Venezuela, Caracas, Venezuela; MHNLS: Museo de Historia Natural La Salle, Caracas, Venezuela; UCLA: Museo de Biología Universidad Centro 


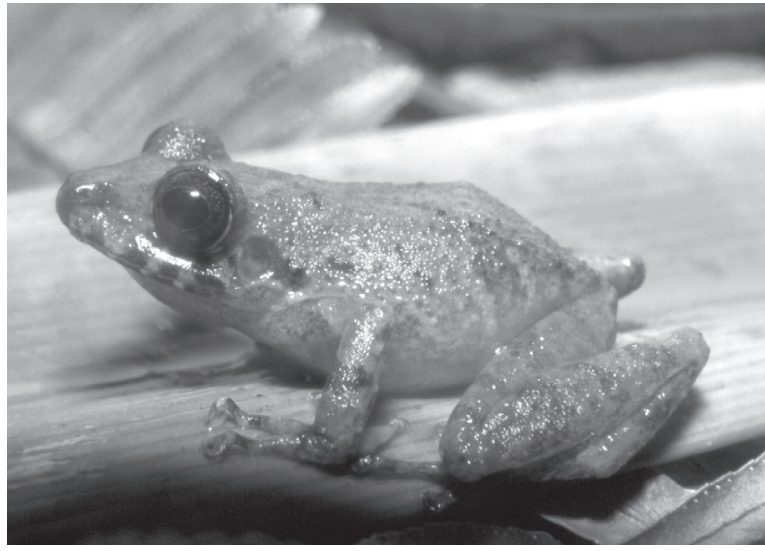

Fig. 1.- Macho de Eleutherodactylus yustizi sp. nov de San Isidro, Barinas.

Fig. 1.- Male of Eleutherodactylus yustizi sp. nov from San Isidro, Barinas.

Occidental Lisandro Alvarado, Barquisimeto, Venezuela; UECA-EE: Unidad de Ecología y Calidad Ambiental, Estación Ecológica Pozo Hondo, DESURCA (Desarrollo Uribante-Caparo), Táchira, Venezuela.

Las comparaciones con material no examinado están directamente tomadas de Lynch (1978) y Rivero (1984a)

La grabación se efectuó con un grabador Sony TCM-353V y un micrófono Sony F-V5, y fue analizada con Cool Edit Pro 1.0 para Windows.

\section{Eleutherodactylus yustizi nov. sp.} (Figs. 1 y 2)

Eleutherodactylus sp.1. Péfaur y Díaz de Pascual, 1987. Rev. Ecol. Latinoamer., 1(3-4): 12.

Eleutherodactylus sp. Yacambú. Yústiz, 1996. Actas II Congr Latinoam. Herpetol. II vol. Publ. U.L.A., C.S.H., Mérida: 347.

Eleutherodactylus tubernasus (parte) Barrio Amorós, 1998. Acta Biol. Venez., 18(2): 55.

Holotipo. CVULA V-2150, un macho adulto de Cerro Alto, Soledad, $8^{\circ} 48^{\prime} \mathrm{N}, 70^{\circ} 31^{\prime} \mathrm{W}$, estado Barinas, Venezuela, $1500 \mathrm{~m}$ aproximadamente, colectada por Jaime Péfaur el 25 de febrero de 1980 .

PARATIPOS. CVULA V-2151, un macho subadulto con la misma localidad, fecha y colector que el holotipo; MBUCV 6602: macho adulto de Yacambú, $1400 \mathrm{~m}$, estado Lara, colectado por O. Fuentes en 2000; CVULA 6704, macho subadulto de la carretera Chavasquén-Córdova, sierra de Portuguesa, $1200 \mathrm{~m}$, estado Portuguesa, sin datos de colector o fecha; EBRG 104950, 1052, 1054-55 y 1058, machos de Cerro el Teteo, 1160 m,

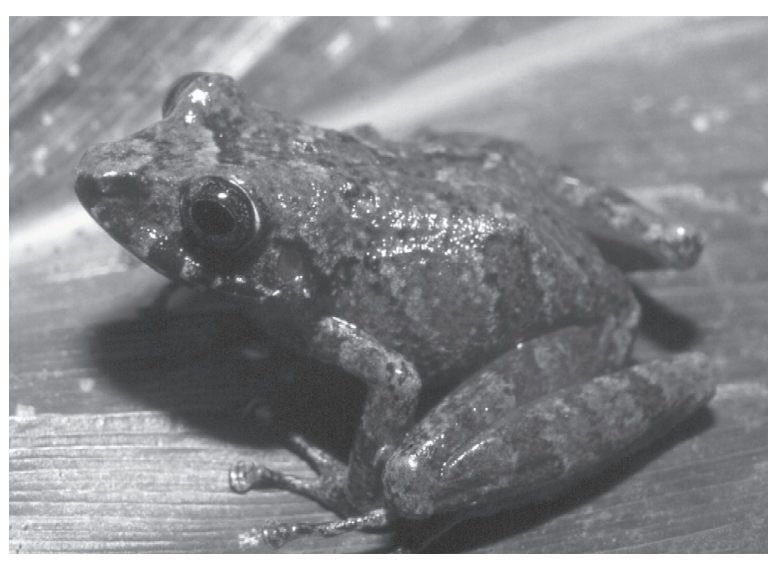

Fig. 2.- Hembra de Eleutherodactylus yustizi sp. nov de Yacambú, Lara.

Fig. 2.- Female of Eleutherodactylus yustizi sp. nov from Yacambú, Lara.

Burgua, entre estados Táchira y Apure, colectados por P. Schwartz el 1 de marzo de 1969; CVULA 6701-3, tres hembras adultas del sendero de interpretación, Parque Nacional Yacambú $\left(9^{\circ} 41^{\prime} \mathrm{N}, 69^{\circ} 28^{\prime} \mathrm{W}\right)$, Serranía de Portuguesa, estado Lara, Venezuela, aproximadamente $1400 \mathrm{~m}$, colectadas por E. Yústiz en fecha indeterminada; UECA-EE-124, hembra adulta de Quebrada Paso Hondo, estado Táchira, colector y fecha indeterminados.

OTRO MATERIAL ESTUDIADO. UECA-EE 461, hembra adulta, Quebrada Caño Negro, estado Táchira, 23-01-1990, colector y fecha indeterminados.

Diagnosis. Un Eleutherodactylus de tamaño moderado (machos hasta $35 \mathrm{~mm}$, hembras hasta $50.8 \mathrm{~mm}$ ), que no situamos en ningún grupo debido a que sus características no concuerdan plenamente con ninguno de los descritos (Lynch \& Duellman, 1997) (ver discusión).

(1) Piel del dorso completamente lisa a finamente granular, la del vientre lisa a granular; (2) tímpano conspicuo pero con contorno poco definido, diámetro aproximadamente un 30-41.5\% del diámetro del ojo; (3) hocico subacuminado a redondeado en vista dorsal, redondeado en perfil; canto rostral bien definido pero suave; (4) APS menor a DIO, con tubérculos apenas visibles, a veces ausentes (ver variación); sin crestas craneales; (5) dientes vomerianos prominentes, oblicuos; (6) presencia de hendiduras vocales y saco gular en machos; (7) primer dedo igual o más corto que el segundo; discos en dedos III y IV mayores que en dedos I y II; discos más anchos que largos; (8) dedos sin rebordes cutá- 


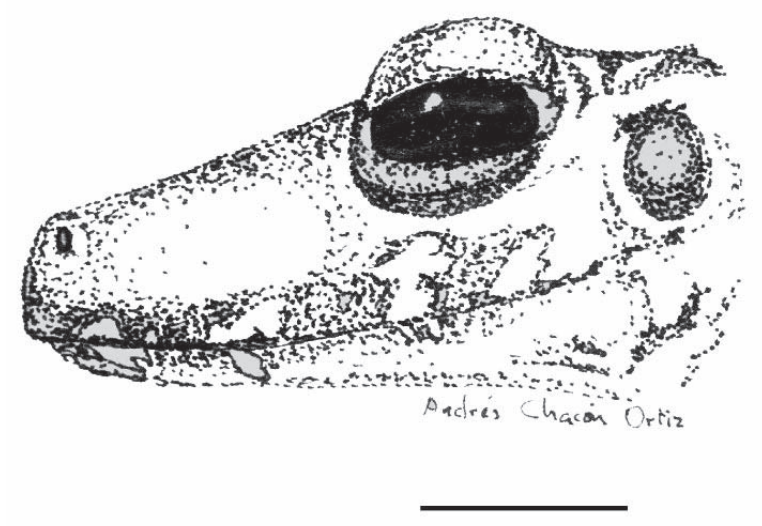

Fig. 3.-Eleutherodactylus yustizi sp. nov. Perfil lateral de la cabeza de CVULA 6703. Escala: $5 \mathrm{~mm}$.

Fig. 3.- Eleutherodactylus yustizi sp. nov. Lateral profile of the head of CVULA 6703. Scale: $5 \mathrm{~mm}$.

neos; (9) tubérculos ulnares ausentes; (10) tubérculos tarsales y calcares ausentes; (11) dos tubérculos metatarsales, el interno oval, de tamaño tres veces mayor que el externo, a veces inconspicuo en machos; (12) dedos del pie palmeados basalmente, sin rebordes cutáneos; ancho del disco del cuarto dedo pedal $80 \%$ a igual que el ancho del disco del tercer dedo manual; (13) dorso castaño casi uniforme, a veces con marcas negras tipo "chevron", en hembras con una $\mathrm{X}$ algo más oscura claramente demarcada por una línea clara que recorre sus bordes (más patente en vida); iris gris verdoso con melanóforos de fondo, con un reticulado negro; anillo periférico ocular azul claro rodeando el iris.

DESCRIPCIÓN. Diez machos, con una LCC máxima de $35 \mathrm{~mm}$, cinco hembras con LCC máxima de $50.8 \mathrm{~mm}$. Cabeza poco más estrecha que ancho del cuerpo (35-39\% de LCC), más larga que ancha; hocico sobresaliendo por encima de los labios, subacuminado en vista dorsal, redondeado en perfil (Fig. 3); narinas no protuberantes, elípticas, dirigidas hacia la parte antero-lateral, área internarial levemente deprimida; canto rostral bien definido pero suave; región loreal plana; labios no sobresalientes; parte superior del párpado con uno o dos pequeños tubérculos apenas notables, a veces ausentes; resto de la cabeza sin tubérculos; APS menor a DIO; ojo grande, prominente, $26.9 \%$ de LCa; pupila elíptica horizontalmente; crestas craneales ausentes; región temporal casi vertical. Pliegue timpánico poco aparente, ocultando el borde supe-
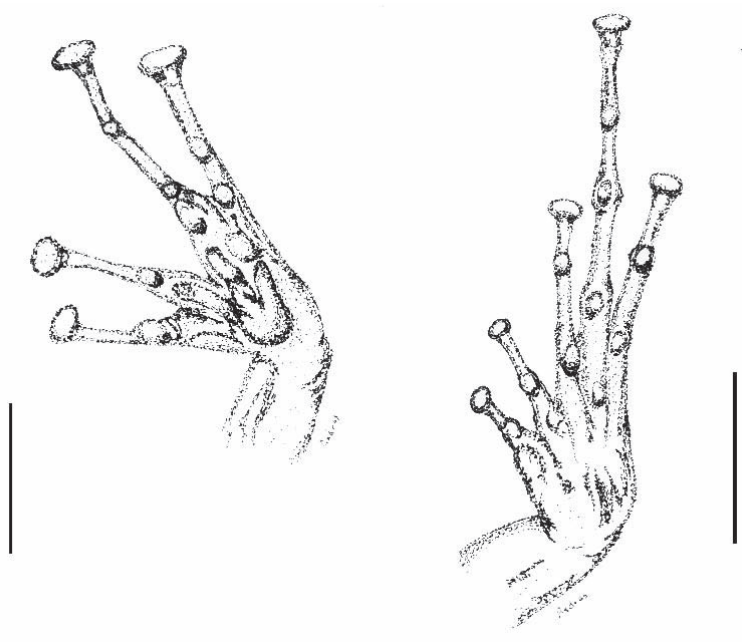

Fig. 4.-Eleutherodactylus yustizi sp. nov. Mano y pie derechos, CVULA 6703. Escala: $5 \mathrm{~mm}$.

Fig. 4.- Eleutherodactylus yustizi sp. nov. Right hand and foot of CVULA 6703. Scale: $5 \mathrm{~mm}$.

rior del anillo timpánico, extendiéndose ligeramente por detrás del tímpano; anillo timpánico visible excepto porción superior; anillo timpánico redondo a oval verticalmente, su longitud $30-47 \%$ del DO, separado del ojo por una distancia poco menor al DT. Coanas ovaladas, pequeñas a medianas, no ocultas por tabique palatal del arco maxilar; procesos vomerianos triangulares, prominentes, oblicuos, separados medialmente, de dos a cinco dientes cada uno; lengua cordiforme, 1/3 libre posteriormente.

Piel del dorso y parte superior de las extremidades posteriores lisa a finamente rugosa, sin espículas, tubérculos ni pliegues; flancos igualmente lisos a rugosos; algunos gránulos mayores bajo el pliegue supratimpánico; a veces un tubérculo prominente postero-inferior al tímpano; sin tubérculos en talón y tarso; cubierta cloacal y tubérculos en región cloacal ausentes; piel de la garganta lisa, del vientre y partes inferiores de las extremidades posteriores lisa a aerolada, a veces granular.

Extremidades anteriores robustas, sin tubérculos ulnares. En la mano, tubérculo tenar oval, ligeramente menor que tubérculo palmar en forma de corazón, bífido; tres a cuatro tubérculos supernumerarios palmares grandes, redondos, sobresalientes, uno en la parte inferior de cada dedo; tubérculos subarticulares grandes, redondos, sobresalientes. Dedos sin rebordes cutáneos; dedo I igual 
o más corto que dedo II; dedos de las manos largos, longitud relativa de dedos $\mathrm{III}>\mathrm{IV}>\mathrm{I}=/ \mathrm{II}$; discos de los dedos más anchos que largos, elípticos, los de dedos III y IV doble ancho que la parte proximal del dedo, todos los discos con almohadillas ventrales (Fig. 4).

Extremidades posteriores largas; talones sobrepasando ampliamente el hocico cuando se flexionan hacia delante, LT 52.3\% de LCC; LP 48.1\% de LCC, sin calcares ni tubérculos o pliegues internos y/o externos del tarso; tubérculo metatarsal interno oval, tres a cuatro veces el tamaño del externo, que es redondo y poco conspicuo; superficies plantares lisas; tubérculos supernumerarios redondos, poco sobresalientes, bajos; tubérculos subarticulares redondos a ovales, sobresalientes. Dedos de los pies largos, delgados, sin rebordes cutáneos, palmeados basalmente; longitud relativa de dedos IV $>$ V $>$ III $>$ II $>$ I; discos de los dedos pedales más pequeños que los de los manuales. Punta del disco del dedo $\mathrm{V}$ sobrepasando penúltimo tubérculo subarticular de dedo IV, punta del disco del dedo III alcanzando el borde inferior del penúltimo tubérculo subarticular.

DiMENSIONES DEL HOLOTIPO. LCC: 31.7; LT: 18.2; LF: 16; LP: 14; ACa: 11.6; LCa: 13; DIN: 3; APS: 3.5 ; DIO: 3.8; DO: 3.7; DT: 1.1; A3D: 1.6; A4D: 1.4; LN: 6; L1D: 5; L2D: 5.

COLORACIÓN EN VIDA. Machos con dorso marrón casi uniforme; sólo algunas marcas negras se distinguen en la parte posterior cervical y escapular. Una barra interorbital gris sucio, bordeada anteriormente por un marrón más claro, casi anaranjado. Barras interlabiales patentes, marrón oscuro, partiendo de la parte inferior del labio superior al ojo. No hay estría cantal; estría post-timpánica delgada, bordeando el límite superior del tímpano. Garganta beige con numerosos melanóforos que le dan un aspecto sucio; pecho rosado con menos melanóforos, vientre beige sucio, con melanóforos abundantes y manchas irregulares de color oro. Antebrazos y tibia con barras transversales marrón oscuro; otras en muslos y brazos mucho menos conspicuas. Iris gris verdoso con melanóforos de fondo, con un reticulado negro. Anillo que rodea el iris azul claro. Según diapositivas de ejemplares no preservados, algunos animales pueden presentar un diseño muy contrastante, con una barra interorbital, estrías cantales y post-timpánicas marrón oscuro a negro y barras en extremidades anteriores y posteriores mucho más contrastadas.

Algunas hembras observadas en Yacambú tenían el dorso pardo brillante, con la referida marca en forma de X claramente contrastada y demarcada por una línea fina mucho más clara, aunque no siempre continua; barras interorbital, supratimpánica y entre labio superior y órbita ocular marrón oscuro; iris bronce, pupila elíptica horizontalmente, negra.

COLORACIÓN EN ALCOHOL. En alcohol, los tres ejemplares hembra de Yacambú son muy similares en diseño y coloración. Dorso de la cabeza y cuerpo pardo oscuro a pardo grisáceo, siendo la parte anterior más oscura que la posterior, con una serie de manchas alineadas formando el contorno de una $\mathrm{X}$ que cubre la totalidad del dorso, más visible hacia los flancos; región loreal marrón muy oscuro; dos barras oscuras conectan el labio con la órbita ocular; una barra inter-orbital muy oscura al nivel de la mitad de los párpados superiores, flanqueada anteriormente por otra barra blanquecina, de menor, igual o mayor grosor que la primera; pliegue timpánico oscuro; tímpano del mismo color que el fondo. Flancos gradualmente más claros hacia el vientre. Superficie dorsal de las extremidades posteriores pardo claro cruzado por barras oscuras transversales, no muy bien definidas en muslos; tres barras bien definidas en la tibia, una o dos en tarsos; superficie posterior de los muslos pardo uniforme, sin ningún tipo de diseño. Superficie ventral pardo grisácea, salpicada irregularmente con pequeñas manchas blanquecinas, que se van difuminando hacia la parte posterior; superficies inferiores de las extremidades posteriores pardo claro casi uniforme, sólo manchadas levemente de blanco; superficies plantar y palmar marrón oscuro. EE-124 es en general muy similar, pero no presenta la barra interorbital, y la $\mathrm{X}$ en el dorso es más delgada y menos definida.

ETIMOLOGíA. Se nomina esta especie en referencia a Enrique Yústiz (in memoriam), gran naturalista del estado Lara, quien declinó la oferta de describir la especie en conjunto. Fue pionero en el estudio de la herpetofauna del estado Lara, y murió de un paro cardíaco en julio de 2003 en Barquisimeto, estado Lara, Venezuela.

DisTRIBUCión. Conocida hasta la fecha de seis localidades diferentes, dos muy próximas en el estado Barinas (apareciendo como una sola en la Fig. 5), tres en el estado Táchira, dos de ellas también muy próximas entre sí (e igualmente representadas en un solo punto en el mapa), una en el estado Lara (Yacambú), y la última en la carretera entre Chavasquén y Córdova en el estado Portuguesa. Entre las dos localidades más distantes, Burgua, en 


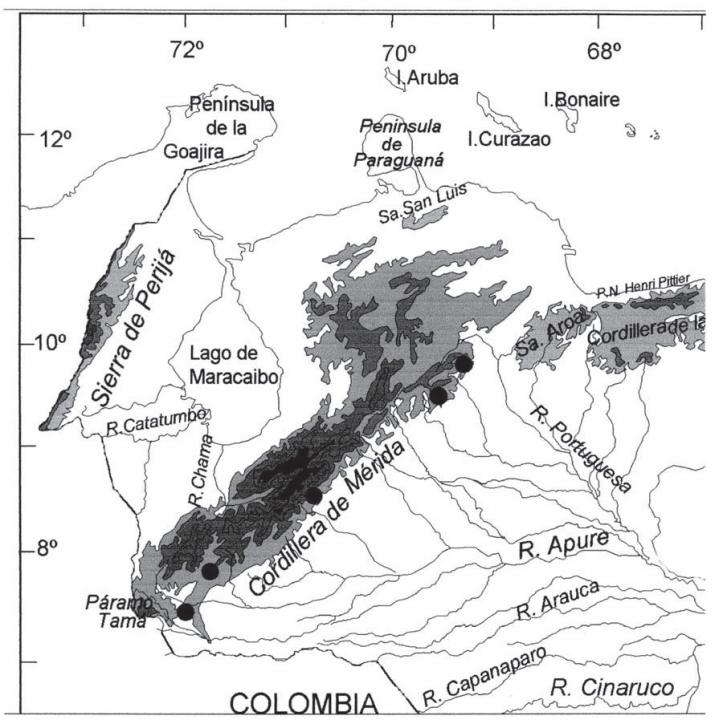

Fig. 5.- Mapa de distribución de Eleutherodactylus yustizi sp. nov. en la cordillera de Mérida de Venezuela.

Fig. 5.- Distribution map of Eleutherodactylus yustizi sp. nov. in the Cordillera de Mérida, Venezuela.

el estado Táchira y Yacambú, en Lara, median 400 $\mathrm{km}$. Eleutherodactylus yustizi se distribuye por todo el piedemonte SE de la Cordillera de Mérida. Las seis localidades pertenecen al ámbito de la selva nublada (bosque muy húmedo premontano de Holdridge) entre 800 y $1600 \mathrm{~m}$. Aunque no ha sido reportado del macizo del Tamá, no sería de extrañar su presencia allá, por la proximidad entre éste y la localidad de Burgua; de esta manera, podría también hallarse en el piedemonte oriental de la Cordillera Oriental de Colombia.

Datos Biológicos. En San Isidro, durante una noche de noviembre de 1995, se observaron varios individuos entre 0.50 y $1.50 \mathrm{~m}$ sobre la vegetación que circundaba un camino amplio y pequeñas quebradas. No se escucharon machos cantando, ni se observó actividad reproductora. La noche del 6 de junio de 2002, sólo se observó un macho posado en una ramita a $20 \mathrm{~cm}$ del suelo. Al rato de ser introducido en una bolsa plástica, inició una serie de cantos que fueron grabados (ver "Vocalización"). Otras especies simpátricas que se observaron en la zona son: un centrolénido (Centrolene o Cochranella) no identificado (en quebradas torrentosas), Hyalinobatrachium sp. (en la misma vegetación que E. yustizi, pero siempre sobre canales de

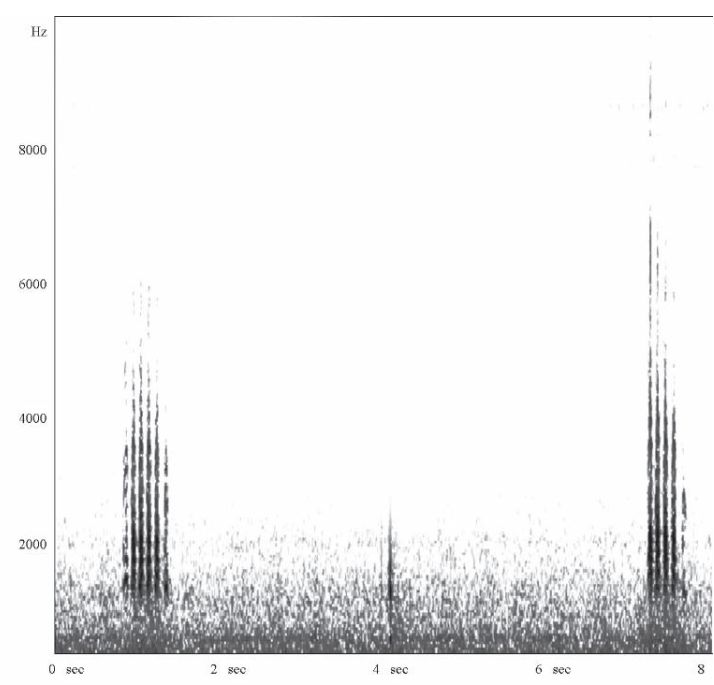

Fig. 6.- Sonograma del canto de Eleutherodactylus yustizi sp. nov. Dos series independientes de 5 y 6 notas cada una, grabadas el 6 de junio de 2002 , sobre las $21.00 \mathrm{~h}$, a $17^{\circ} \mathrm{C}$, en San Isidro, estado Barinas.

Fig. 6.- Sonogram of two independent series of calls of Eleutherodactylus yustizi sp. nov., one of 5 and other of 6 notes, recorded on 6 June 2002 , about $21.00 \mathrm{~h}, 17^{\circ} \mathrm{C}$, at San Isidro, Estado Barinas.

agua corriente); Eleutherodactylus chlorosoma (un solo ejemplar sobre una hoja de gran tamaño) y Gastrotheca nicefori Gaige, 1933 (una hembra que se capturó durante el día sobre el suelo en el mismo lugar donde se iba a levantar la tienda de campaña, y que se liberó tras fotografiar). En el Parque Nacional Yacambú, E. yustizi ha sido hallado sobre hojas de arbustos entre los 0.5 y los $2 \mathrm{~m}$.

VoCALIZACIÓN. El canto que hemos grabado proviene de un ejemplar encerrado temporalmente en una bolsa plástica. Nunca escuchamos el canto al natural, por lo que no sabemos si se trata de un canto nupcial o de otra índole. Es un canto elaborado, en series de cinco a seis notas (Fig. 6) durando $450 \mathrm{~ms}$ ( 5 notas) o $500 \mathrm{~ms}$ (6 notas). Las primeras notas de cada secuencia duran $60 \mathrm{~ms}$. La frecuencia dominante es en ambos casos de $2200 \mathrm{~Hz}$, mientras que la frecuencia fundamental es de $1200 \mathrm{~Hz}$ tanto en la primera serie como en la segunda. La temperatura durante la grabación es de $17^{\circ} \mathrm{C}$.

OBSERVACIONES. Una fotografía de esta especie aparece bajo el nominativo común de "ranita de niebla" en una publicación divulgativa (Barrio, 2000). 
COMPARACIÓN CON OTRAS ESPECIES. Eleutherodactylus yustizi se diferencia del resto de las especies de Eleutherodactylus presentes en selvas nubladas de la Cordillera de Mérida (entre 900 y $2500 \mathrm{~m}$ ) por las siguientes características (entre paréntesis las de E. yustizi). De las especies del complejo tubernasus [E. chlorosoma, E. pulidoi y E. tubernasus] por presentar éstas un tubérculo o prominencia en la punta del hocico (sin tubérculo), tímpano grande, la mitad o 3/4 del diámetro del ojo (pequeño, nunca llegando a la mitad del diámetro del ojo), y color dorsal en vida verde (marrón con variantes, pero nunca verde). De las especies del complejo lentiginosus [E. melanoproctus, E. mondolfii, $E$, vanadisae, E. lentiginosus] por presentar éstas manchas amarillas sobre fondo rojo o rosado en las partes ocultas de los muslos e ingles (sin manchas amarillas ni partes ocultas como las descritas), carecer de hendiduras vocales, excepto $E$. lentiginosus (presentes), presencia de tubérculos ulnares y tarsales (ausentes). De E. pleurostriatus por poseer éste tímpano grande, $2 / 3$ del diámetro del ojo (pequeño, menor a $1 / 2$ ), primer dedo mucho más corto que el segundo (igual), tubérculos ulnares mínimos (ausentes), y ausencia de hendiduras vocales (presentes). De E. prolixodiscus por presentar éste almohadillas en los discos de las manos más largos que anchos, y almohadilla del dedo IV redonda (más anchos que largos). Las especies de páramo (por encima de los $2500 \mathrm{~m}$ ), E. boconoensis, E. briceni, E. colostichos E. ginesi, E, lancinii, E. paramerus, son de complexión robusta y compacta, y poseen las extremidades posteriores cortas, nunca superando la punta del hocico cuando se flexionan de manera perpendicular al eje del cuerpo (superándola siempre en E. yustizi). De E. pedimontanus porque éste presenta la piel dorsal granular (finamente granular); membrana timpánica prominente, que cubre 40 a $80 \%$ del diámetro del ojo (tímpano conspicuo pero con contorno poco definido, diámetro aproximadamente un 30-41.5\% del diámetro del ojo); hocico truncado en vista lateral (redondeado); 12 dientes vomerianos en cada proceso (2-5); dedos de la mano con repliegues laterales y gruesos (ausentes); tubérculos ulnares poco conspicuos (ausentes).

Eleutherodactylus yustizi se acerca en su diagnosis al grupo unistrigatus (sensu Lynch \& Duellman, 1997), pero se diferencia (características del grupo unistrigatus en paréntesis) en que el párpado superior es más estrecho que la distancia interorbital (generalmente tan ancho como, o más ancho), piel ventral lisa a finamente granular (areolada), dedo I de la mano igual o ligeramente más corto que II (dedo I más corto que II), tubérculos subarticulares moderadamente prominentes (no prominentes), membrana pedal basal entre III y IV, y IV y V (dedos de los pies generalmente sin rastro de membrana), y dedo $\mathrm{V}$ del pie sobrepasa tubérculo subarticular penúltimo pero no alcanza tubérculo distal (dedo $\mathrm{V}$ del pie considerablemente más largo que dedo III, y con punta del disco generalmente alcanzando el tubérculo distal del dedo IV).

Eleutherodactylus yustizi tampoco cabe en el grupo conspicillatus (sensu Lynch \& Duellman, 1997) (caracteres diferenciales del grupo conspicillatus entre paréntesis) por su dedo I igual o ligeramente más corto que II (generalmente dedo I más largo que II), tubérculos subarticulares moderadamente prominentes (no prominentes), varios tubérculos supernumerarios palmares prominentes (pocos o ausentes), tarso sin pliegues (muchas especies del grupo con pliegue interno tarsal), dedos del pie palmeados basalmente (sólo una especie de 29 con pies palmeados basalmente, $E$. malkini).

\section{Discusión}

En la Cordillera de Mérida se conocen sólo dos especies pertenecientes al grupo unistrigatus, E. mondolfii y E. colostichos (Lynch \& Duellman, 1997). Curiosamente, muchas otras especies de este mismo ramal andino carecen de asignación a un grupo concreto de especies. Así, Lynch \& Duellman (1997) sólo reconocen el grupo tubernasus de Rivero (1984a), pero no asignan E. pleurostriatus, E. boconoensis, E. melanoproctus, E. vanadisae, E. briceni, E. ginesi, E. paramerus y E. lentiginusus, a ningún grupo de especies, lo cual no deja de ser indicativo de que falta mucho por estudiar de las especies venezolanas. Eleutherodactylus pedimontanus pertenece al grupo conspicillatus, siendo hasta la fecha el único representante de este grupo en la Cordillera de Mérida. De todas formas, E. yustizi no pertenece claramente al grupo unistrigatus ni al grupo conspicillatus por las diferencias que hemos mencionado anteriormente.

Otras especies del género habitan también en Venezuela, principalmente en áreas montañosas fuera de los Andes, como la Cordillera de la Costa (11 especies) y los relieves del Escudo Guayanés (incluyendo zonas bajas amazónicas: 10 especies), totalizando 38 especies, si no contamos con una especie introducida, E. johnstonei Barbour, 1914, que está ampliando su distribución continuamente (Kaiser et al. 2002). A pesar de la aparente riqueza 
en especies del género (el más diverso en Venezuela), ésta es muy baja si se compara con la de su vecino Colombia, con 210 especies conocidas (Acosta-Galvis, 2000), y con la de un país mucho más pequeño como es Ecuador, con 136 especies (Guayasamín, 2004), gracias en gran parte a la muy activa dedicación del Dr. John D. Lynch en ambos países. Otro hecho discriminante es que tanto en Colombia como en Ecuador especies de zonas bajas ascienden hasta alturas considerables a través de los valles andinos, cosa que al parecer no ocurre en Venezuela (Rivero, 1984b). Venezuela, no obstante, posee muchas zonas completamente inexploradas en lo que a presencia de especies de Eleutherodactylus se refiere. Creemos, concretamente, que tanto en las laderas y cimas de los tepuis de la Guayana venezolana como en las extensas áreas montañosas, especialmente páramos y selvas nubladas, poco o nada exploradas en la Cordillera de Mérida y Sierra de Perijá debe de haber no pocas especies esperando ser descubiertas y descritas.

\section{AgRAdecimientos}

El primer autor desea agradecer a Andrés Orellana su determinante compañía en el campo y la hospitalidad de su familia durante la estancia en Barquisimeto. Sobre todo, queremos agradecer a Enrique Yústiz, quien nos atendió diligentemente, nos donó sus datos personales, y nos cedió la posibilidad de describir la especie que, en honor a su interés, le dedicamos. A los curadores de los museos visitados, Amelia Díaz de Pascual (CVULA), Ramón Rivero (EBRG), Mercedes Salazar (MBUCV) y Celsa Señaris (MHNLS). Marcelo Molinillo (INFOGEO, PAT, Mérida) elaboró el mapa que usamos. Rafael Aguilar nos ayudó a analizar el canto.

\section{Referencias}

Acosta-Galvis, A. R. 2000. Ranas, Salamandras y Caecilias (Tetrapoda: Amphibia) de Colombia. Biota Colombiana, 1(3): 289-319.

Barrio, C. L., 2000. Ranas en Apuros. El País Semanal, 7 de mayo 2000: 47-49.

BARRIO-AmORÓs, C. L., 1998. Sistemática y Biogeografía de los anfibios (Amphibia) de Venezuela. Acta Biológica Venezuelica, 18(2): 1-93.

Duellman, W. E., 1992. A new species of the Eleutherodactylus conspicillatus group (Anura: Leptodactylidae) from Northeastern Peru. Revista Española de Herpetología, 6: 23-29.

Fuentes, O. \& Barrio-Amorós, C. L. A new Eleutherodactylus (Anura, Leptodacvtylidae) from Marahuaka tepui, Amazonas, Venezuela. Revista Colombiana de Ciencias. En prensa.
Guayasamín, J. M., 2004. A new species of Eleutherodactylus (Anura: Leptodactylidae) from the northwestern lowlands of Ecuador. Herpetologica, 60(1): 103-116.

Kaiser, H., Barrio-Amorós, C. L., Trujillo, J. \& LYNCH, J. D., 2002. Expansion of Eleutherodactylus johnstonei in Northern South America: Rapid Progress Through Human Interactions. Herpetological Review, 33(4): 290-294.

Kaiser, H., Hardy, J. D. \& Green, D. M., 1994. Taxonomic status of Caribbean and South American frogs currently ascribed to Eleutherodactylus urichi (Anura: Leptodactylidae). Copeia, 1994(3): 780-796.

LA Marca, E., Descripción de dos nuevos anfibios del piedemonte andino de Venezuela. Herpetotrópicos, 1(1): $1-9$

LYNCH, J. D., 1978. A new Eleutherodactylinae frog from the Andes of northern Colombia (Leptodactylidae). Copeia, 1978(1): 17-21.

LYNCH, J. D., 1999. Lista anotada y clave para las ranas (género Eleutherodactylus) chocoanas del Valle del Cauca, y apuntes sobre las especies de la Cordillera Occidental adyacente. Caldasia, 21(2): 184-202.

LyNCH, J. D. \& Duellman, W. E., 1997. Frogs of the genus Eleutherodactylus (Leptodactylidae) in Western Ecuador: Systematics, Ecology and Biogeography. The University of Kansas Natural History Museum, Special Publication, 23: 1-236.

Rivero, J. A., 1964. Salientios (Amphibia) en la colección de la Sociedad de Ciencias Naturales La Salle. Caribbean Journal of Science, 4(1): 297-305.

Rivero, J. A., 1984a. Los Eleutherodactylus (Amphibia, Leptodactylidae) de los Andes Venezolanos II. Especies subparameras. Memoria de la Sociedad de Ciencias Naturales La Salle, 42(118): 57-132.

Rivero, J.A., 1984b. Sobre las relaciones y el origen de los Eleutherodactylus (Amphibia: Leptodactylidae) andinos de Venezuela. Caribbean Journal of Science, 20(3-4): 139-144.

RuedA, J. V. \& LyNCH, J. D., 1983. Una nueva especie de Eleutherodactylus (Amphibia: Leptodactylidae) para la Cordillera Oriental de Colombia. Lozania (Acta Zoologica Colombiana), 42: 1-6.

YúsTIZ, E., 1996. Aspectos biogeográficos de la herpetofauna de la cuenca hidrográfica del río Turbio (Estado Lara, Venezuela). In: Péfaur. J. E. (ed.) Herpetología Neotropical. Actas del II Congreso Latinoamericano de Herpetología. II vol. Publ. U.L.A., C.S.H. Mérida: 317-349. 
Apéndice.- Material examinado.

Appendix.- Material examinado.

\section{VENEZUELA:}

Eleutherodactylus briceni: CVULA 2752-56, Páramo de la Culata, estado Mérida.

Eleutherodactylus ginesi: CVULA 5817-21: Mucubají, estado Mérida.

Eleutherodactylus lancinii: CVULA 0621, 0995-98, 1000 , 1007-08, 1010-11, 1993, 1995-96, 5826-27: Páramo de Mucubají, estado Mérida.

Eleutherodactylus paramerus: CVULA 0766, 0992-93, 1003 04, 1859, 5828, 5830-34, 5836, 5839-46, 5848, 5850 5853: Páramo de Mucubají, estado Mérida.

Eleutherodactylus vanadisae: CVULA 0744-47, 3108-20, 3123-27: La Mucuy, Parque Nacional Sierra Nevada, estado Mérida, Venezuela.

Eleutherodactylus "urichi”: MHNLS 409, Sierra de Périja.

\section{COLOMBIA:}

Eleutherodactylus megalops: CVULA 3095-96: Magdalena. Santa Marta, Serranía; San Lorenzo, Sierra.

Eleutherodactylus nicefori: CVULA 5558-60: El Alto, km 5 , carretera Capilla-Cocuy, Boyacá.

Eleutherodactylus w-nigrum: CVULA 3097-98: Cauca: Páez, km 34 Belcázar-Tacuayo. 